\title{
The Post-Secondary Student Stressors Index (PSSI): Proof of Concept and Implications for Use
}

\author{
Brooke Linden ${ }^{1}$, Randall Boyes ${ }^{2}$, and Heather Stuart ${ }^{1-2}$ \\ ${ }^{1}$ Health Services and Policy Research Institute, Queen's University, Kingston, ON, Canada \\ ${ }^{2}$ Department of Public Health Sciences, Queen's University, Kingston, ON, Canada
}

\begin{abstract}
Objective: This study demonstrates the utility of the Post-Secondary Student Stressors Index (PSSI), an instrument designed to identify and evaluate the sources of student stress. The PSSI is comprised of 46 stressors, rated by severity and frequency, across five domains: academics, learning environment, campus culture, interpersonal, and personal.

Participants: Pilot testing of the tool was conducted among $n=535$ post-secondary students enrolled at an Ontario university.

Methods: Mean severity and frequency ratings were calculated for each stressor on the instrument. Results were plotted, stratifying results by sex. T-tests for differences in means across sexes were calculated for each stressor.

Results: Female students in this sample consistently rated nearly all stressors on the instrument as more severe than their male counterparts. Females also reported higher frequency ratings on average, indicating that they worried more often about stressors than males. Domain-specific stressors are discussed.

Conclusions: The PSSI can provide post-secondary institutions with the ability to target and improve their mental health promotion and mental illness prevention efforts.
\end{abstract}

Keywords: post-secondary; student; mental health; stress; health promotion 


\section{Background}

In recent years, chronic stress and poor mental health among students have become major concerns for post-secondary institutions across Canada ${ }^{1,2}$ and elsewhere ${ }^{3-5}$. Excessive stress among students has been linked to numerous negative outcomes, including poor mental health ${ }^{6}$ and interference with academic performance ${ }^{7}$. The 2016 iteration of the National College Health Assessment II (NCHA II) survey revealed excessive stress and symptoms consistent with common mental illnesses among Canadian post-secondary students, with the crude prevalence estimates for self-reported symptoms of past-year anxiety and depression increasing between the 2013 and 2016 iterations of the survey ${ }^{7,8}$. Evidence of severe distress, including self-harm (8.7\%), suicidal ideation (13\%), and previous suicide attempts $(2.1 \%)$, were also prominent, sparking significant concern about the wellbeing of this population.

While large-scale efforts to monitor the prevalence of mental health problems among post-secondary students are available (e.g., the NCHA II survey) or are in development (e.g., the Canadian Campus Wellbeing Survey, currently in development at the University of British Columbia ${ }^{9}$ ), each comes with their own limitations. For example, the NCHA II survey was designed primarily to evaluate physical health characteristics and behaviours. As a result, the mental health measures included on the survey are limited and have been met with strong criticisms ${ }^{10}$. While students are asked to share the overall level of stress they have experienced within the past 12-month period, as well as the degree to which stress has impacted their academic performance, more specific questions regarding the sources of this stress are notably absent. The NCHA II does touch upon some common sources of stress with a series of questions inquiring about factors affecting academic performance (e.g., work, roommate difficulties, health concerns, injury, etc.) but these are not always specific to the student experience. Additionally, the decision to place each of these factors only in context of how they have impacted a student's academic performance is problematic, given that previous research has shown some of the highestperforming students that exhibit characteristics such as perfectionism to be the most at risk for chronic stress and mental health-related challenges ${ }^{11-13}$. In contrast, the Canadian Campus Wellbeing Survey fills some of the gaps left by the NCHA II by focusing exclusively on student mental health and wellbeing. However, stress remains only one component of the relatively large subject area of "wellbeing", and as such, is not as comprehensive an assessment as institutions may be need to make informed decisions regarding the tailoring of their mental health promotion and mental illness prevention programming on campus based on the stressors students experience.

The Post-Secondary Student Stressors Index is not the first instrument designed to evaluate the sources of post-secondary student stress, with several instruments having been created in the 1990's and early 2000 's to address this area of inquiry ${ }^{14-16}$. However, in addition to being outdated, many of these instruments are associated with a number of issues that preclude them from being useful, including 
scope-related issues, poor psychometric properties, and evaluation of only one dimension of stress (e.g., most frequently only severity of stress). The PSSI was designed to fill the gaps left by its predecessors.

The PSSI includes stressors from across multiple domains of the student experience, including: academics, the learning environment, campus culture, the interpersonal, and the personal. The instrument was developed using an explanatory sequential mixed methods research design conducted over a two-year period, placing emphasis on a "for-students-by-students" approach. Students were actively engaged in the development, refinement, and testing of the tool, and treated as subject matter experts. Using a combination of qualitative and quantitative methods, the authors sought to not only identify a holistic list of the sources of student stress, but also to understand the reasons why these things prompted a stress reaction in students ${ }^{17}$. The ability to more accurately identify the sources of student stress is the first step towards helping post-secondary institutions to develop efficacious mental health promotion and mental illness prevention efforts and maximize their effectiveness for their student body.

\section{Objectives}

The objectives of this study were: 1) to present proof-of-concept, demonstrating the utility of the PSSI; and 2) to offer recommendations for the future use of the tool. We conducted a sex-based analysis of stressors, to observe whether male and female students' experiences of stress differed within the postsecondary setting. The findings presented in this paper lead to recommendations applicable to this student body (e.g., how services might be targeted given the data observed), and serve as a guideline for other institutions that may be interested in utilizing the PSSI.

\section{Methods}

\section{Sample and Participants}

The data presented here were collected from a sample of students at a mid-sized Ontario university that was also used to facilitate the collection of evidence for validity. A random sample of 5000 students was provided by the Office of Institutional Research and Planning. All enrolled students were eligible to participate, with no exclusion criteria applied. A total of 535 participants completed the survey, representing a response rate of $11 \%$. Although low, this is somewhat typical of response rates for student surveys, and sufficient for our purposes here. All participants consented to participate in the online survey. This research received ethics clearance from the institutional ethics board.

\section{Measures}

\section{Post-Secondary Student Stressors Index}

The PSSI is comprised of 46 stressors across five major domains of stress: academics, the learning environment, campus culture, the interpersonal, and the personal. Students are asked to indicate the 
severity of stress they experience ("How stressful is this?") for each item on the instrument, as well as the frequency with which this stress occurs ("How often are you stressed about this?"). Response options ranged from a scale of 1 ('not stressful' and 'rarely') to 4 ('very stressful' and 'almost always'), with an additional option to indicate N/A in the event that a stressor was not applicable. The PSSI has been evaluated for preliminary validity and reliability, demonstrating strong psychometric properties ${ }^{18}$.

\section{Demographics}

In addition to the PSSI, participants were asked to provide demographic information, including: sex, year of birth, relationship status, living arrangement during the academic year, level of study, student status, estimated grade point average, and whether or not they were an international student.

\section{Analysis}

Descriptive statistics were calculated for the demographic characteristics of the sample. We calculated the mean severity and mean frequency for each stressor on the instrument, identifying the top stressors in each domain. If a participant indicated either that they did not consider an item to be stressful or that the item never happened to them (and by extension, rated frequency as not applicable), they were not included in these mean scores. The proportion of people included in the averages on this basis can be observed in Appendix A, Table A-1. Mean scores were plotted for both male and female students on two-by-two plots, stratified by severity and frequency. This visual display facilitates easy observation of the priority areas for each domain of stress (e.g., those that are above average in both severity and frequency). Plots were developed for each domain of stress, as well as one larger, overall plot depicting all stressors measured by the PSSI. Finally, t-tests for differences in means across sexes were conducted for both severity and frequency scores for each stressor (reported in Appendix B, Table B-1).

\section{Results}

\section{Demographics}

Demographic characteristics of the sample are reported in Table 1. Most participants were female (74.0\%), single (64.9\%), lived off campus with roommates $(62.1 \%)$, self-reported their GPA to be between $80-89 \%$ (41.7\%), and studied full-time (92.1\%) and at the undergraduate level (65.5\%). The majority of participants were between the ages of 19 and 21 years (63.7\%), with an overall average age of 24.5 years $(S D=7.0)$. International students made up about $9 \%$ of the sample. 
Table 1. Demographic Characteristics of Sample $(n=535)$

\begin{tabular}{|c|c|c|}
\hline Variable & $\begin{array}{c}\text { Frequency } \\
n\end{array}$ & $\begin{array}{c}\text { Percent } \\
\%\end{array}$ \\
\hline \multicolumn{3}{|l|}{ Sex } \\
\hline Female & 396 & 74.0 \\
\hline Male & 132 & 24.7 \\
\hline Non-Binary & 3 & 0.6 \\
\hline Prefer not to answer & 4 & 0.7 \\
\hline \multicolumn{3}{|l|}{ Age Group } \\
\hline $18-21$ years & 264 & 49.3 \\
\hline $22-25$ years & 130 & 24.3 \\
\hline $26-29$ years & 65 & 12.1 \\
\hline $30+$ years & 76 & 14.2 \\
\hline \multicolumn{3}{|l|}{ Relationship Status } \\
\hline Single & 347 & 64.9 \\
\hline In a Relationship & 111 & 20.7 \\
\hline Married or Common-law & 68 & 12.7 \\
\hline Separated, Divorced, or Widowed & 2 & 0.3 \\
\hline Prefer not to answer & 7 & 1.3 \\
\hline \multicolumn{3}{|l|}{ Living Arrangement } \\
\hline Campus residence hall & 35 & 6.4 \\
\hline Other on-campus housing & 13 & 2.4 \\
\hline Off campus with roommates & 332 & 62.1 \\
\hline Off campus alone & 69 & 12.9 \\
\hline Off campus with family & 83 & 15.5 \\
\hline Prefer not to answer & 3 & 0.6 \\
\hline \multicolumn{3}{|l|}{ Level of Study } \\
\hline $1^{\text {st }}-2^{\text {nd }}$ year Undergraduate & 187 & 34.9 \\
\hline $3^{\text {rd }}-5^{\text {th }}$ year Undergraduate & 154 & 28.8 \\
\hline Graduate (Masters) & 72 & 13.5 \\
\hline Graduate (Doctoral) & 53 & 9.9 \\
\hline Professional Program & 47 & 8.8 \\
\hline Other & 21 & 3.9 \\
\hline Prefer not to answer & 1 & 0.2 \\
\hline \multicolumn{3}{|l|}{ Student Status } \\
\hline Full-time & 493 & 92.1 \\
\hline Part-time & 32 & 6.0 \\
\hline Other ${ }^{\mathrm{a}}$ & 8 & 1.5 \\
\hline Prefer not to answer & 2 & 0.4 \\
\hline \multicolumn{3}{|l|}{ International Student } \\
\hline No & 486 & 90.8 \\
\hline Yes & 48 & 9.0 \\
\hline Prefer not to answer & 1 & 0.2 \\
\hline \multicolumn{3}{|l|}{ Approximate GPA } \\
\hline $90-100 \%$ & 103 & 19.3 \\
\hline $80-89 \%$ & 223 & 41.7 \\
\hline $70-79 \%$ & 147 & 27.5 \\
\hline $60-69 \%$ & 32 & 6.0 \\
\hline $0-59 \%$ & 4 & 0.7 \\
\hline Prefer not to answer & 26 & 4.9 \\
\hline
\end{tabular}

Note. Valid percents reported.

a "Other" category includes non-degree seekers, online students, medical residents. 


\section{Overall Results}

First, we observed the mean severity and frequency ratings for stressors within each domain. Academic stressors consistently ranked among the most severe stressors, but the frequency with which they were experienced varied. Certain stressors (such as thesis projects, practicums, and meetings with advisors) were rated as severe, but reported by fewer students. This is to be expected, given the relatively smaller proportion of students in the sample to whom these stressors were likely to apply (e.g., graduate students, students in professional programs). Stressors within the learning environment were generally rated as infrequent and less severe. Among stressors related to campus culture, a range of frequency and severity ratings were observed. Sexual harassment was reported infrequently, even among those reporting it ever, but was rated as severely stressful when it did occur. Interpersonal stressors included the stressor reported to occur most frequently across the entire sample: pressure to own expectations. All stressors in the personal category were rated above average in frequency, but severity scores ranged from among the lowest (health-related stressors such as cooking, exercise, nutrition, and sleep) to among the highest (financial pressures, including having to take student loans, and worrying about getting a job after graduation). Figure 1 depicts the mean severity and frequency ratings for all stressors included in the instrument.

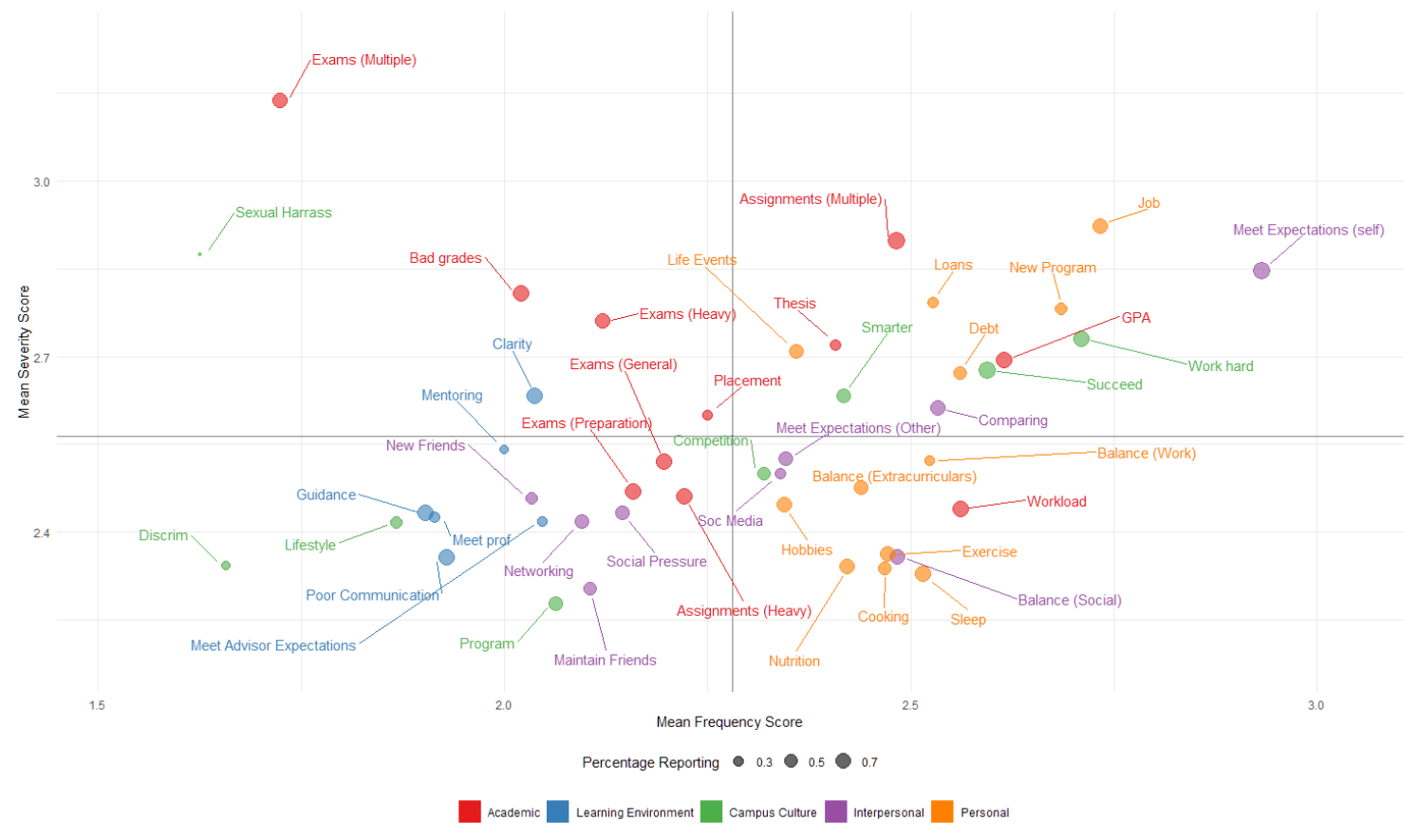

Figure 1. Mean severity and frequency ratings for all stressors PSSI (total sample)

\section{Sex-Based Analysis}

Within each domain of stress, the mean severity and frequency scores for stressors were plotted against each other for male (blue) and female (red) participants (Figures 1-5). A dotted line connects the male and female scores for each stressor. The size of the point is proportional to the number of participants who were affected by the stressor, with larger points reflecting higher frequencies. The axes on the plot 
represent the overall mean severity and frequency for all stressors in the domain of interest (excluding those who rated severity as 0 [not applicable] or 1 [not stressful]), as rated by the entire sample. T-tests were conducted to assess whether the severity and frequency scores reported by male and female students were significantly different for each stressor (see Appendix B).

Overall, female students' most severe stressors were having multiple exams in the same week, writing heavily weighted exams (i.e., worth $50 \%+$ of final course grade), worrying about getting into a new program, taking loans, incurring debt, and meeting their own expectations. Their most frequent stressors were meeting their own expectations, pressure to succeed, debt, loans, and having multiple assignments to complete simultaneously. The most commonly reported stressors by female students were multiple assignments (96\%), heavy workload (93\%), and meeting their own expectations (92\%), while their least frequently reported were working on thesis (28\%), and lack of mentoring from supervisor (28\%), and meeting supervisor's expectations (30\%). Male students' most severe stressors were having multiple exams, worrying about getting a job, having multiple assignments due at the same time, sexual harassment on campus, and meeting their own expectations. Their most frequent stressors were meeting their own expectations, worrying about getting a job, worrying about not work hard enough, worrying about getting into a new program, and maintaining a high GPA. The most commonly reported were meeting their own expectations (89\%), multiple assignments $(89 \%)$, and pressure to succeed $(84 \%)$. The least commonly reported were sexual harassment (12\%), discrimination (27\%), and lack of mentoring from supervisor $(28 \%)$.

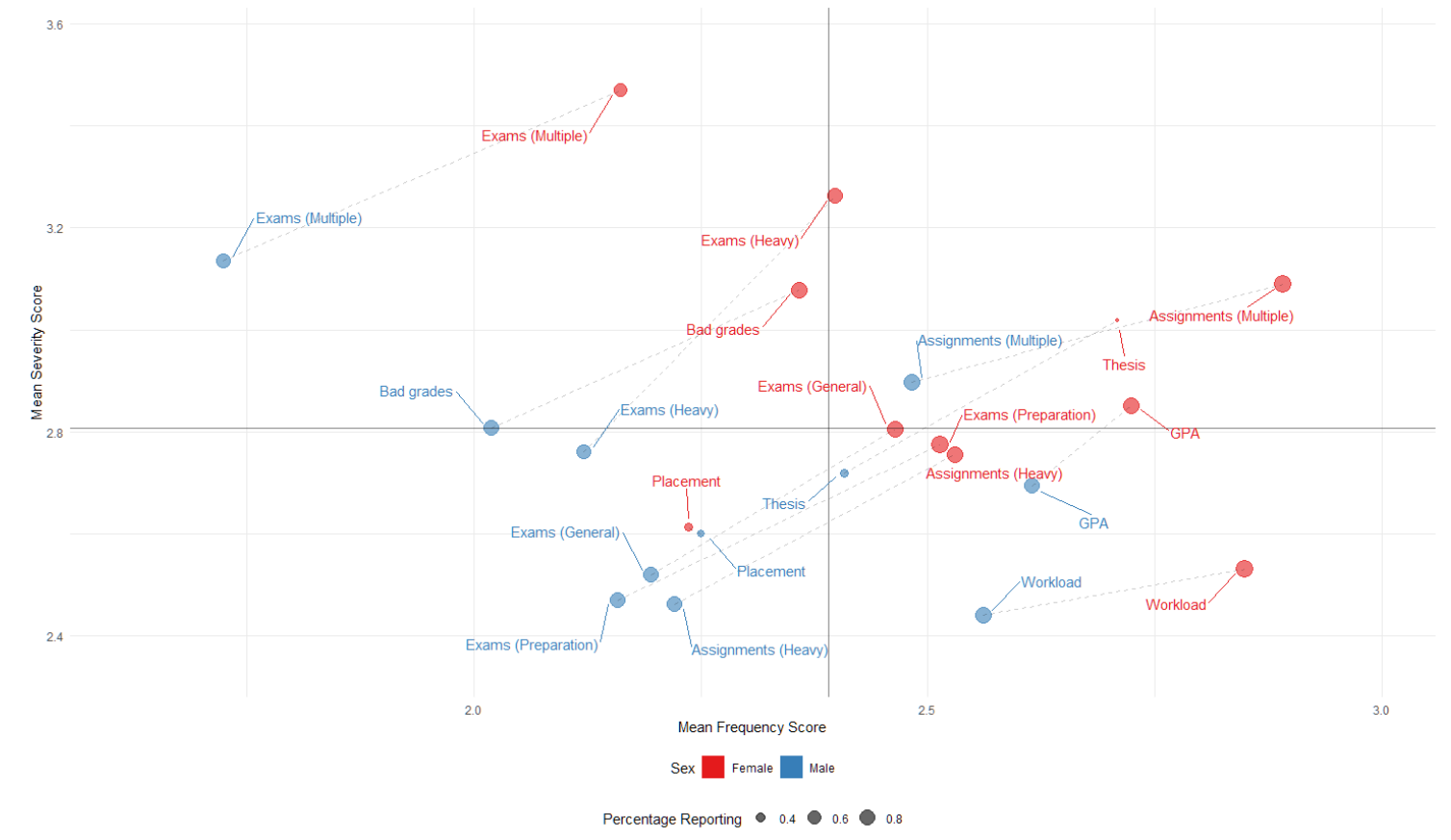

Figure 2. Mean severity and frequency ratings for academic stressors, by sex 


\section{Academic Domain}

On average, female (F) students reported academic stressors to be more severe and more frequent than did male (M) students, suggesting that female students more often worry about academic stressors than male students. The largest absolute differences in mean severity by sex were observed for: exams worth $50 \%$ or more $(\mathrm{F}+0.50 \%)$, having multiple exams in one week $(\mathrm{F}+0.33 \%)$, preparing for exams ( $\mathrm{F}$ $+0.30 \%$ ), and working on thesis ( $\mathrm{F}+0.30 \%$ ). By mean frequency, the largest differences were observed for: having multiple exams in one week $(\mathrm{F}+0.44 \%)$, having multiple assignments due around the same time $(\mathrm{F}+0.41 \%)$, and preparing for exams $(\mathrm{F}+0.35 \%)$, and receiving a bad grade $(+0.34 \%)$. The smallest difference for both mean severity and frequency ratings between sexes was observed for performing well at placement $(\mathrm{F}+0.01 \%,-0.01 \%)$. Significant mean differences by sex for both severity and frequency were observed for nearly all stressors in this domain, with the exceptions of managing academic workload, maintaining GPA, and performing well at placement.

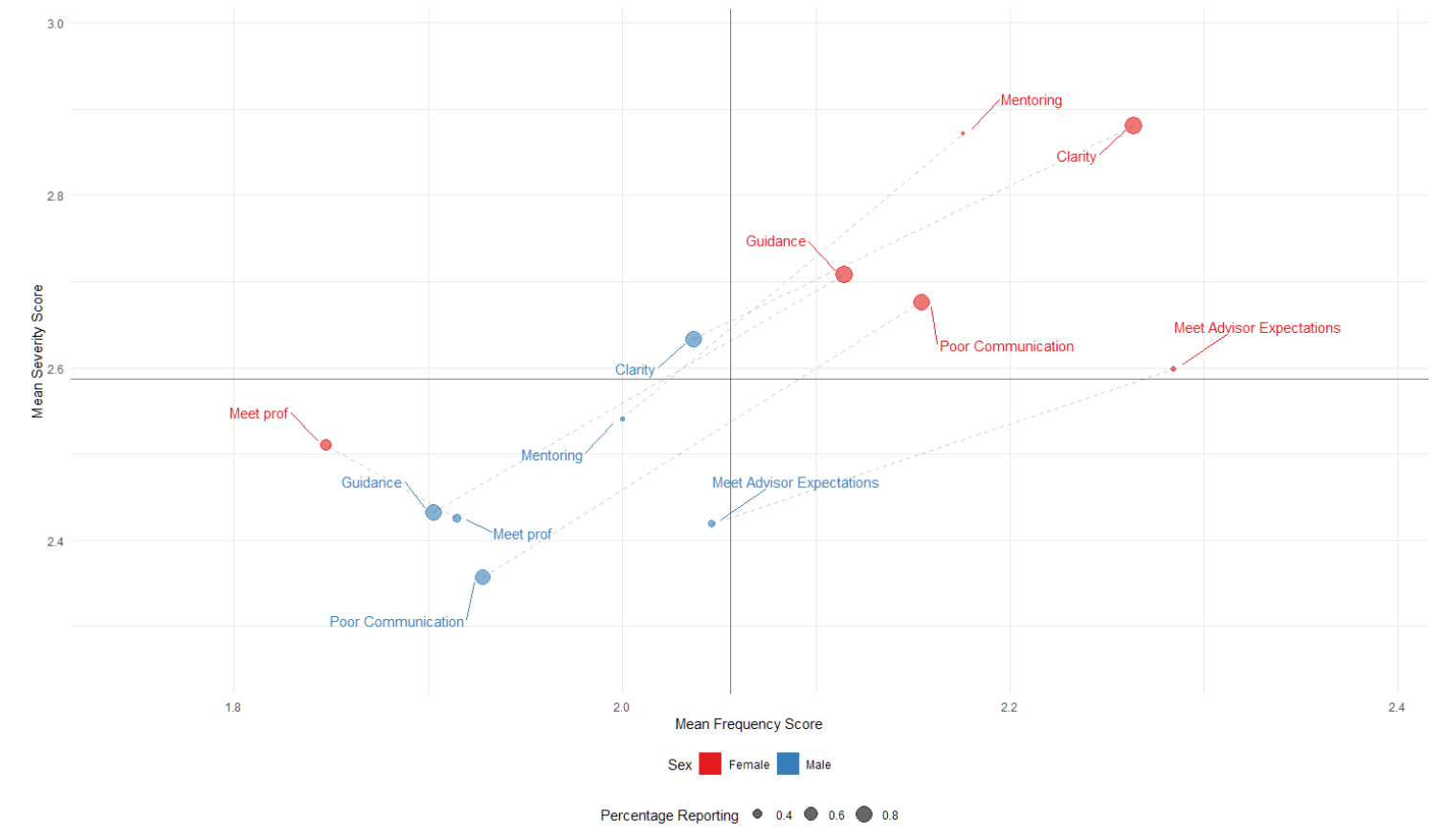

Figure 3. Mean severity and frequency ratings for learning environment, by sex

\section{Learning Environment Domain}

Female students generally reported stressors within the learning environment to be more severe and more frequently occurring than their male counterparts. The largest absolute differences in mean severity by sex were observed for: lack of mentoring from supervisor $(\mathrm{F}+0.33 \%)$, poor communication from professor $(\mathrm{F}+0.32 \%)$, and lack of guidance from professor $(\mathrm{F}+0.28 \%)$. For frequency, the largest differences were for: meeting advisor's expectations $(\mathrm{F}+0.23 \%)$, poor communication from professor $(\mathrm{F}+0.22 \%)$, lack of clarity from professor $(\mathrm{F}+0.22 \%)$, and lack of guidance from professor $(\mathrm{F}+0.21 \%)$. The smallest difference for both severity and frequency was reported for meeting with professor, with female students reporting the average severity to be slightly higher $(+0.08 \%)$ compared to male 
students, but the average frequency to be slightly lower (-0.06\%). Significant differences in both mean severity and frequency were observed across sexes for poor communication, lack of clarity, and lack of guidance, with a significant difference in mean severity also observed across sexes for lack of mentoring from advisor.

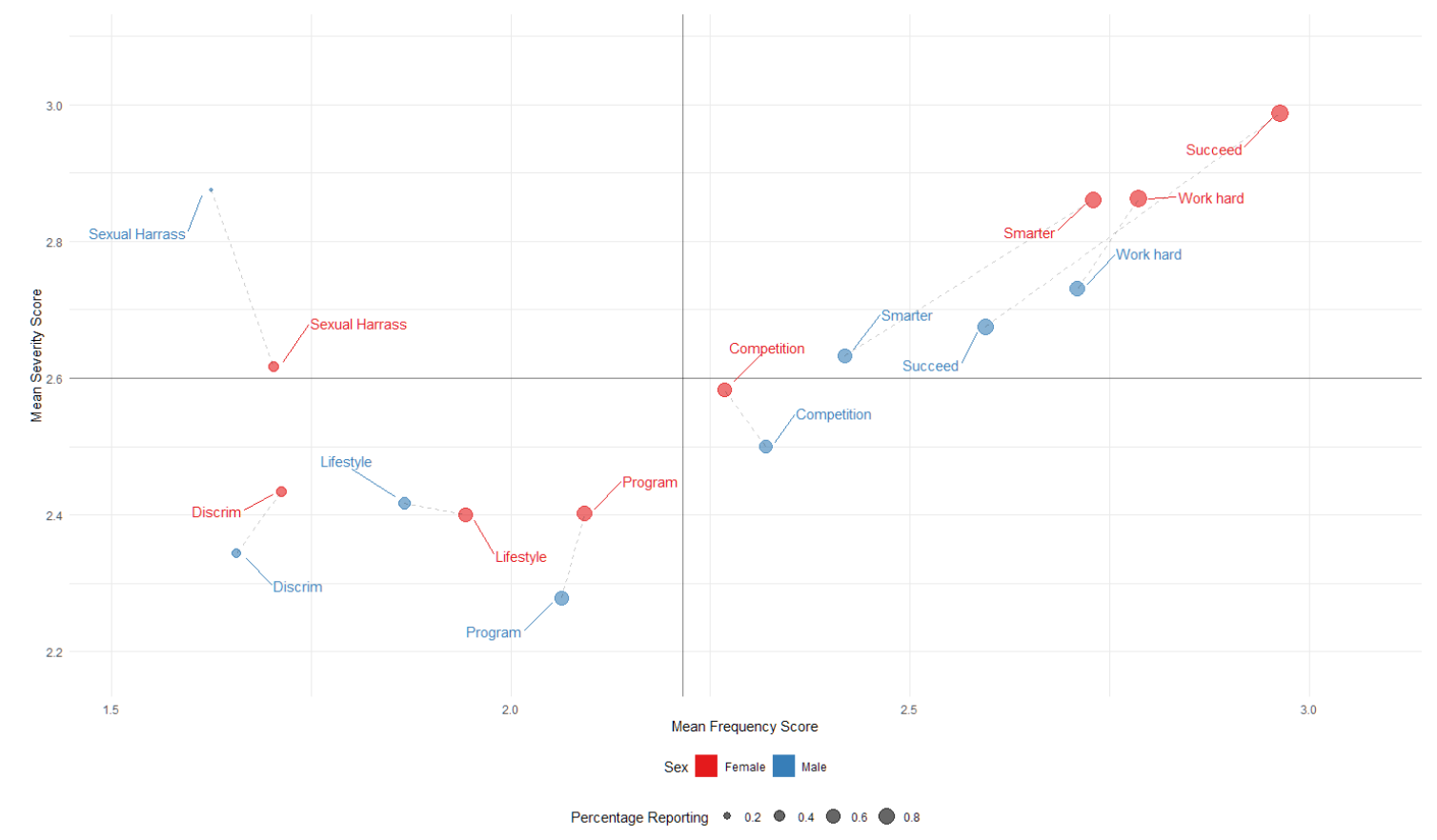

Figure 4. Mean severity and frequency ratings for campus culture, by sex

\section{Campus Culture Domain}

Results for stressors within the campus culture domain were variable. In general, female students tended to report these stressors as being more severe compared to male students, with the exception of sexual harassment on campus. While a larger proportion of female students reported sexual harassment on campus ( $32 \%$ compared to $12 \%$ of males), the average severity rating provided by female students for this stressor was lower than that provided by their male counterparts ( $\mathrm{F}-0.26 \%)$, despite females reporting this stressor more frequently $(\mathrm{F}+0.08 \%)$. The largest absolute differences in mean severity by sex were observed for: pressure to succeed ( $F+0.31 \%)$, sexual harassment on campus $(M+0.26 \%)$, and feeling like my peers are smarter than I am $(\mathrm{F}+0.23 \%)$. By frequency, the largest differences were observed for: pressure to succeed $(\mathrm{F}+0.37 \%)$ and feeling like my peers are smarter than I am (F $+0.31 \%$ ). The smallest difference in severity was observed for adjusting to the post-secondary lifestyle, with female students' rating this stressor as slightly less severe than male students (F - $0.02 \%$ ), while the smallest difference in frequency was observed for adjusting to a new program $(\mathrm{F}+0.03 \%)$. Only two statistically significant differences in mean severity and frequency were observed across sexes in this domain: feeling like my peers are smarter than I am, and pressure to succeed. 


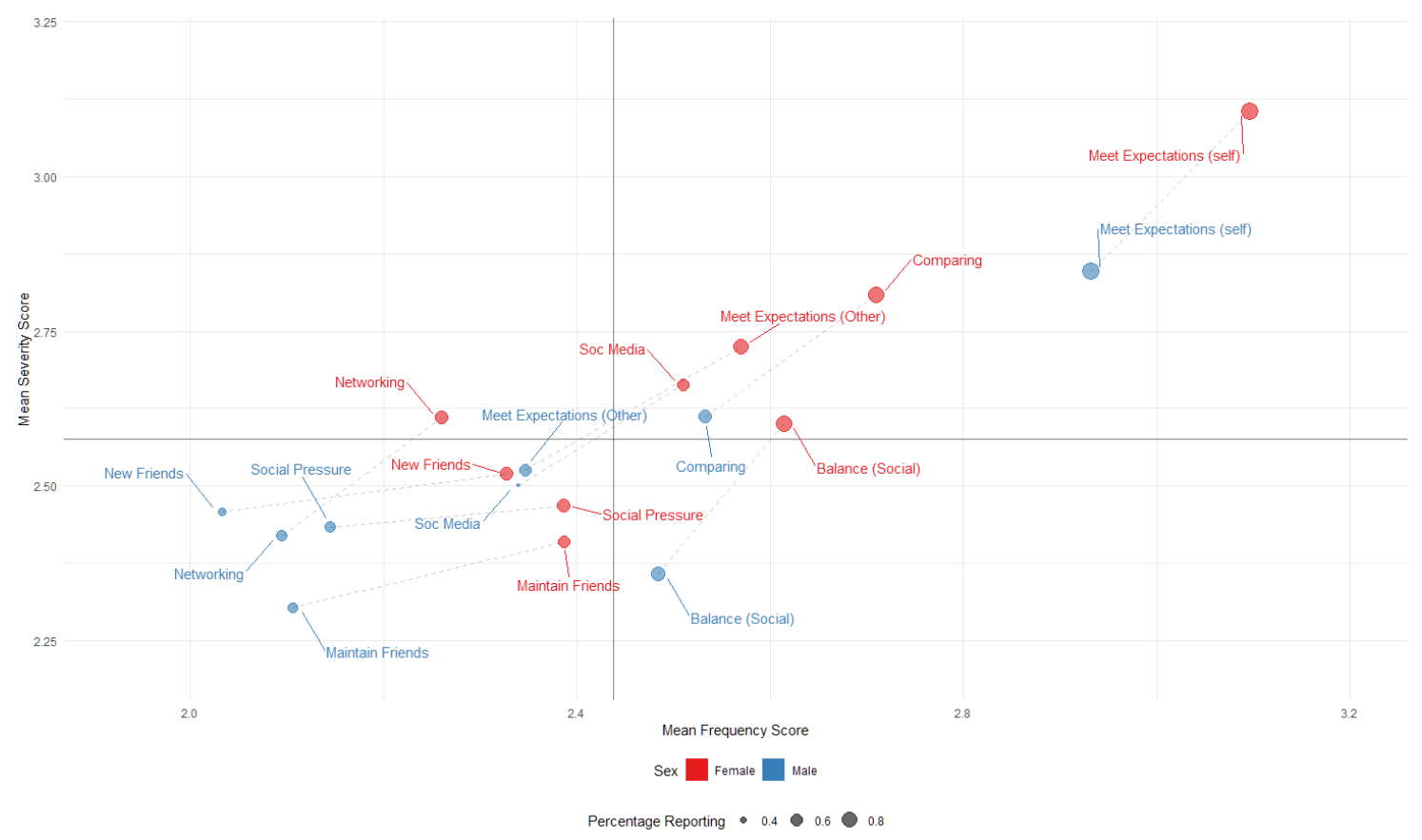

Figure 5. Mean severity and frequency ratings for interpersonal stressors, by sex

\section{Interpersonal Domain}

Female students consistently reported interpersonal stressors as being more severe compared to male students, with the largest absolute differences in mean severity by sex observed for: meeting own expectations $(\mathrm{F}+0.25 \%)$, balancing social life with academics $(\mathrm{F}+0.24 \%)$, networking $(\mathrm{F}+0.19 \%)$, and meeting others' expectations ( $\mathrm{F}+0.19 \%)$. For frequency, the largest differences were observed for: making new friends $(\mathrm{F}+0.30 \%)$, maintaining friendships $(\mathrm{F}+0.28 \%)$, pressure to socialize ( $\mathrm{F}+0.28 \%)$, and meeting others' expectations $(\mathrm{F}+0.22 \%)$. The smallest difference in severity was observed for pressure to socialize $(\mathrm{F}+0.04 \%)$, while the smallest difference in frequency was observed for balancing a social life with academics $(\mathrm{F}+0.13 \%)$. Significant differences in mean severity across sexes were observed for networking, balancing social life with academics, comparing myself to others, and meeting expectations (both others' and self). Significant differences in mean frequency across sexes were observed for making new friends, maintaining friendships, pressure to socialize, and meeting others' expectations. 


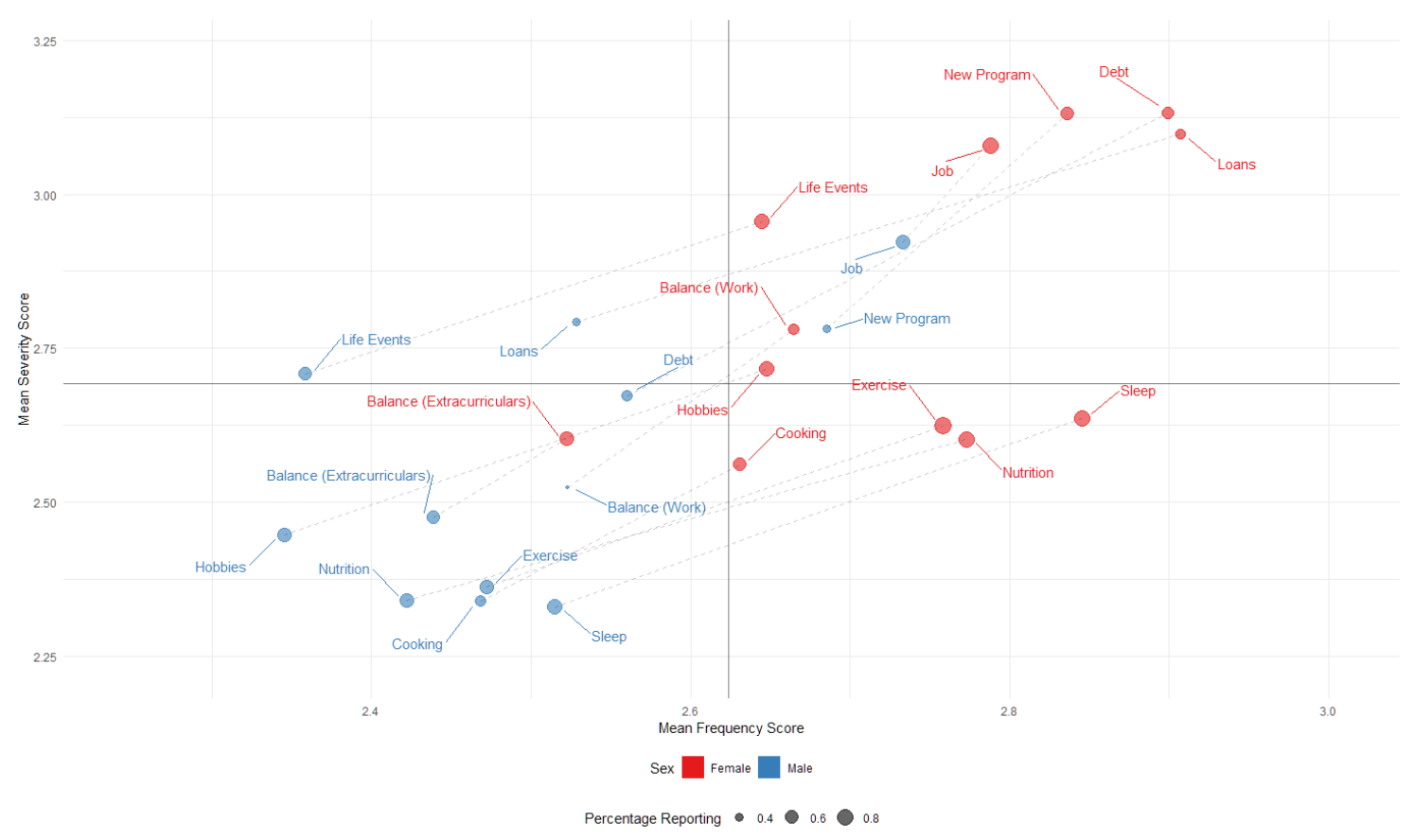

Figure 6. Mean severity and frequency ratings for personal stressors, by sex

\section{Personal Domain}

Female students consistently reported personal stressors as being more severe and more frequent in occurrence than their male counterparts. The largest absolute differences in mean severity by sex were observed for: debt $(\mathrm{F}+0.46 \%)$, worrying about getting into a new program $(\mathrm{F}+0.35 \%)$, having to take student loans $(\mathrm{F}+0.31 \%)$, and getting enough sleep $(\mathrm{F}+0.31 \%)$. For frequency, the largest differences were observed for: loans $(\mathrm{F}+0.38 \%)$, nutrition $(\mathrm{F}+0.35 \%)$, and debt $(\mathrm{F}+0.34 \%)$. The smallest difference in mean severity was observed for balancing extracurriculars with academics $(\mathrm{F}+0.12 \%)$, while the smallest difference in mean frequency was observed for worrying about getting a job after graduation $(F+0.06 \%)$. Significant mean differences in severity across sexes were observed for every stressor in this domain, with the exceptions of balancing extracurriculars with academics and worrying about getting a job. No significant differences in frequency across sexes were observed for cooking, balancing work with academics, balancing extracurriculars with academics, worrying about getting a job, or worrying about getting into a new program post-graduation.

\section{Discussion}

The purpose of developing the PSSI was to provide a better assessment of students' exposure to stressors within the post-secondary setting. In this paper, proof-of-concept for the utilization of the tool was demonstrated among a sample of students at a mid-sized university in Ontario. Female students in this sample consistently rated nearly all stressors on the instrument as more severe than their male counterparts. Females also reported higher frequency ratings on average, indicating that they worried more often about stressors than males. This is consistent with existing knowledge regarding female 
students' tendency to self-report a higher degree of stress and distress than male students ${ }^{7}$. Results within each domain of stress are discussed below in context of existing literature.

With respect to academic stressors, female students consistently reported higher mean severity ratings by an average of about $+0.30 \%$, compared to male students. Female students also tended to report higher frequencies in this category, indicating that females worried about academic stressors more often than their male counterparts. Some of the most severe stressors for both sexes included managing multiple exams or assignments within a short time frame, while managing the academic workload was the most frequently reported stressor. Together, these findings suggest that that time management may be a key component of students' academic stress, consistent with previous literature. Indeed, previous research has found the management of academic demands to be one of the most commonly cited sources of stress for students ${ }^{19-21}$. One recommendation for this particular institution might be to provide students with the tools and education required to improve their ability to multitask as part of their mental health promotion activities. In addition, they may consider policy options that prevent multiple exams within a short time frame (e.g., no more than two exams in a five-day period).

Secondly, writing heavily weighted exams worth $50 \%$ or more was identified as the second most severe stressor for both sexes, and affected a large proportion of the sample. This is consistent with existing literature identifying test anxiety as a major academic stressor for many students ${ }^{22}$. Moving forward, the implementation of a policy restricting finals to a maximum weighting of $40 \%$ may be warranted. Recent discussions within the Scholarship of Teaching and Learning have begun to explore the utility of a paradigmatic shift in the assessment of student learning, moving away from standardized tests and examinations as a method of assessment towards more practical alternatives such as assignments and oral presentations ${ }^{23}$. It is possible that a shift away from exams as a primary method of assessment may have a positive impact on reducing student stress.

Within the learning environment domain, female students reported communication and clarity issues with faculty members as more severe and frequently stressful in comparison to male students. This may be linked to female students' comparatively greater concern for achieving academic excellence compared to males, as observed within the academic domain. Previous research has shown that constructive student-faculty interactions are connected to several positive outcomes for students, including feelings of belongingness on campus, positive academic self-concept, and positive psychosocial wellbeing, highlighting the importance of these interactions to students' overall wellbeing ${ }^{24}$. One exception to this rule was the item "meeting with professors," for which female students reported the lowest severity and frequency ratings. This might indicate that female students are more comfortable reaching out to professors for help than their male counterparts. This is in line with previous research which has found that females reach out to faculty members, particularly with requests about 
coursework, more often than male students ${ }^{24,25}$. One policy change that might be explored at this institution is improved education and awareness for faculty and staff regarding the resources available to students who may be struggling with their coursework (e.g., student academic success services, writing centres). Additionally, many institutions offer brief courses through their Centres for Teaching and Learning that may be beneficial for faculty and staff in determining how to improve or clarify their curricula and expectations of students.

Findings in the campus culture category were mixed. Both males and females reported a desire to excel, with "pressure to succeed," "feeling like I'm not working heard enough," and "feeling like my peers are smarter than I am" leading the board in terms of both severity and frequency. Females rated pressure to succeed as being the most severe and frequent stressor in this domain, while males rated concerns about not working hard enough as having the greatest impact on their stress levels. Male students found competition with their peers to be more frequently stressful than females, tough females found this stressor to be slightly more severe. The severity of these stressors may reflect this particular institution's reputation as a high-achieving, research-based, top university in Canada. These stressors are frequently noted symptoms of "imposter syndrome," a condition where an individual feels they are fraudulent, or undeserving of their accomplishments and responsibilities, despite having earned them. Given the negative mental health effects imposter syndrome can produce, a possible recommendation might be to provide (or increase) education for students about imposter syndrome, and how to manage and/or combat it as a component of mental health promotion programming ${ }^{26}$.

The identification of these stressors within the campus culture is consistent with previous literature that has highlighted the impact of environmental factors on students' overall wellbeing ${ }^{27}$. In fact, campus culture has been consistently linked to students' psychological health and wellbeing, with negative perceptions or experiences predicting less favourable health outcomes ${ }^{28}$. In particular, student experiences of racism, sexism, or other forms of discrimination and/or harassment have been linked to poor mental health outcomes ${ }^{29,30}$. Among this sample of students, experiences of sexual harassment and discrimination on campus were fairly rare, though rated as relatively severe when they did occur. Interestingly, male students reported sexual harassment to be both more severe and frequently occurring than female students. This may be explained by the wording of the item ("sexual harassment on campus") which was made intentionally general so as to not only invite responses from individuals who had experienced (or been a victim of) sexual harassment, but also those who witnessed it occurring on campus. Institutions should continue to enforce a no-tolerance policy regarding instances of sexual harassment and discrimination, in addition to continuing to educate students about campus resources available to them following these experiences. 
The majority of the stressors within the interpersonal domain were rated similarly in terms of severity and frequency, with females' ratings slightly higher. Male students rated pressure to socialize as being a more frequent stressor than females. This is consistent with what male students shared during the development phase of research for the PSSI ${ }^{17}$ as well as with existing literature regarding the pressure male students feel to meet the social expectations of what it means to be a male university or college student (e.g., often in the context of alcohol consumption and "partying") ${ }^{31,32}$. Despite pressure to socialize being a salient stressor for male students, making new friends and maintaining friendships were rated as less severe and less frequently stressful in comparison. One study found that the most frequent presenting issues for Canadian post-secondary students seeking counselling revolved around relationship concerns ${ }^{19}$. Perhaps additional mental health promotion programming that focuses on relationship management should be made available to students, particularly to those who may be more vulnerable to these stressors (e.g., first-year undergraduates).

Female students tended to rate social media as more severe and frequently stressful than male students. Though there has been little exploration into the impact of social media on post-secondary students' mental health and wellbeing to date, research among younger, adolescent populations suggests that this may be an important issue for incoming cohorts of students over the next several years. The most substantial stressor in this domain for both males and females was "meeting my own expectations," with both males and females rating this stressor well above average severity, and females rating it both more severe and frequently occurring than males. It is possible that the pressures produced by social media's frequent outward displays of perfectly "tailored" lives are contributing towards students placing pressure on themselves to meet unattainable expectations. Institutions should explore whether introducing education around healthy online (e.g., social media) habits into their mental health promotion programming may mitigate these stressors.

Within the personal domain, financial concerns were the most substantial stressors for female students, including incurring debt, having to take student loans, and worrying about getting a job after graduation. Male students also reported that worrying about getting a job was a relatively severe stressor (albeit, slightly less severe than females), but concerns regarding debt and loans were much lower by both severity and frequency. Financial concerns have long been acknowledged in the literature as a primary source of stress among student populations ${ }^{33,34}$. This suggests a need for institutions' health promotion programming to include financial literacy resources for students. Female students also tended to report health-related stressors (e.g., exercise, nutrition, getting enough sleep) as being more severe and frequent than male students. Previous research has found that healthful behaviours, such as getting enough sleep, engaging in regular physical activity, and eating a balanced diet have been linked to reductions in stress ${ }^{35}$ and improved wellbeing ${ }^{36}$. The largest difference between sexes in this domain was observed for worrying about reaching major life events, which is consistent with existing literature 
on sex-based concerns about reaching milestones, such as marriage and having children, particularly among graduate students ${ }^{37}$. Institutions might consider refocusing their mental health promotion programming to encompass elements of both physical and mental health, given the evolving definition of mental health and "wellness" as encompassing both physical and mental health components ${ }^{38,39}$.

\section{Limitations}

Despite its strengths, there are some limitations to this research. Our analysis was based on a moderately small sample of students at a single Ontario university, which is unlikely to be representative of Canada's broader post-secondary student population. We strongly recommend that institutions interested in using this tool to make policy changes to mental health services on campus make every effort to gather a representative sample of their student body to ensure that inferences drawn from the sample are valid. Secondly, while the PSSI has been shown to be valid among post-secondary students in Ontario, little is known about its generalizability to student populations in other regions of Canada, or other countries such as the United States of America. Future research involving the PSSI should include a multi-site validation assessment across multiple universities in varying regions of Canada. Until the validity of the tool is evaluated in other settings and among different populations, it should be used with caution.

\section{Conclusions}

The PSSI assesses 46 stressors across five major domains of stress specific to the post-secondary student experience: academics, the learning environment, campus culture, the interpersonal, and the personal. The PSSI's dual-evaluation of both the severity and frequency of stress facilitates an analysis that can provide institutions with a straightforward method of pinpointing the most severe and frequently occurring stressors on their campus. This knowledge will not only facilitate the identification of initial priority areas for improvement, but will also allow institutions to more effectively target their mental health promotion and mental illness prevention programming.

\section{Contributor Notes}

BL is a Postdoctoral Research Fellow with the Health Services and Policy Research Institute at Queen's University in Kingston, Ontario, Canada. RB is a PhD Candidate in the Department of Public Health Sciences. HS is a Professor in the Department of Public Health Sciences at Queen's University, and the Bell Canada Mental Health and Anti-Stigma Research Chair.

\section{Conflicts of Interest}

The authors declare that they have no conflicts of interest to disclose. 


\section{References}

1. Holmes A, Silvestri R. Rates of mental illness and associated academic impacts in Ontario's college students. Can J Sch Psychol. 2016;31(1):27-46. doi:10.1177/0829573515601396

2. Council of Ontario Universities. Foundations: Mental Health and WellBeing Initiatives at Ontario's Universities. Toronto; 2017. https://cou.ca/.

3. Stewart-Brown S, Evans J, Patterson J, et al. The health of students in institutes of higher education: an important and neglected public health problem? J Public Health Med. 2000;22(4):492-499.

4. Stallman H. Prevalence of psychological distress in university students: implications for service delivery. Aust Fam Physician. 2008;37(8):673-677.

5. Bernhardsdottir J, Vilhjalmsson R. Psychological distress among university female students and their need for mental health services. $J$ Psychiatr Ment Health Nurs. 2013;20(8):672-678.

doi:10.1111/jpm.12002

6. Eisenberg D, Gollust SE, Golberstein E, Hefner JL. Prevalence and correlates of depression, anxiety, and suicidality among university students. Am J Orthopsychiatry. 2007;77(4):534-542. doi:10.1037/00029432.77.4.534

7. American College Health Association. American College Health Association National College Health Assessment II: Canadian Reference Group Data Report Spring 2016. Hanover, MD; 2016. http://www.acha-ncha.org/.

8. American College Health Association. American College Health Association National College Health Assessment II: Canadian Reference Group Data Report Spring 2013. Hanover, MD; 2013.

9. Faulkner G, Ramanathan S, Kwan M. Summary: Delphi Results and Roundtable Discussions for the Development of the Canadian Campus Wellbeing Survey. Vancouver; 2018.

10. Rahn RN, Pruitt B, Goodson P. Utilization and limitations of the American College Health Association's National College Health Assessment instrument: a systematic review. J Am Coll Heal. 2016;64(3):214237. doi:10.1080/07448481.2015.1117463

11. Hu KS, Chibnall JT, Slavin SJ. Maladaptive Perfectionism, Impostorism, and Cognitive Distortions: Threats to the Mental Health of Pre-clinical Medical Students. Acad Psychiatry. $\quad$ February 2019. doi:10.1007/s40596-019-01031-z

12. Newman BN, Strickler JG, O'Brien C, Lui $\mathrm{T}$, Lynch M. Deconstructing perfectionism in college students: Patterns of behavior, emotion, and cognition. Pers Individ Dif. 2019;145:106-111.

doi:10.1016/j.paid.2019.03.030

13. Shannon A, Goldberg JO, Flett GL, Hewitt PL. The relationship between perfectionism and mental illness stigma. Pers Individ Dif. 2018;126(November 2017):66-70. doi:10.1016/j.paid.2018.01.022

14. Ross S, Niebling B, Heckert T. Sources of stress among college students. Coll Stud J. 1999;33(2):312.

15. Rocha-Singh IA. Perceived stress among graduate students: development and validation of the Graduate Student Stress Inventory. Educ Psychol Meas. 1994;54(3):714-727.

16. Towbes LC, Cohen LH. Chronic stress in the lives of college students: Scale development and prospective prediction of distress. J Youth Adolesc. 1996;25(2):199217.

17. Linden B. Understanding Post-Secondary Student Stress: A Qualitative Analysis. June 2019.

https://advance.sagepub.com/articles/Under standing_Post-

Secondary_Student_Stress_A_Qualitative Analysis/8233562.

18. Linden B, Stuart H. Psychometric Assessment of the Post-Secondary Student Stressors Index (PSSI). BMC Public Health. 2019;19:1139. doi: /10.1186/s12889-0197472-z

19. Cairns SL, Massfeller HF, Deeth SC. Why do postsecondary students seek counselling? Can J Couns. 2010;44(1):34-50.

20. Kruisselbrink Flatt A. A suffering generation: six factors contributing to the mental health crisis in North American higher education. Coll Q. 2013;16(1):17.

21. Robinson AM, Jubenville TM, Renny K, 
Cairns SL. Academic and mental health needs of students on a Canadian campus. Can J Couns Psychother. 2016;50(2):108123.

22. Zeidner M. Test Anxiety. In: The Wiley Handbook of Anxiety Disorders. Chichester, UK: John Wiley \& Sons, Ltd; 2014:581595. doi:10.1002/9781118775349.ch28

23. Gipps C. Beyond Testing (Classic Edition). 1 st ed. London: Routledge; 2011. doi:10.4324/9780203182437

24. Cohen ED. Gendered styles of studentfaculty interaction among college students. Soc Sci Res. 2018;75(October 2017):117129. doi:10.1016/j.ssresearch.2018.06.004

25. Kim YK, Sax LJ. Student-faculty interaction in research universities: Differences by student gender, race, social class, and first-generation status. Res High Educ. 2009;50(5):437-459. doi:10.1007/s11162-009-9127-x

26. Ramsey E, Brown D. Feeling like a fraud: Helping students renegotiate their academic identities. Coll Undergrad Libr. 2018;25(1):86-90. doi:10.1080/10691316.2017.1364080

27. Brook CA, Willoughby T. The social ties that bind: social anxiety and academic achievement across the university years. $J$ Youth Adolesc. 2015;44(5):1139-1152. doi:10.1007/s10964-015-0262-8

28. McBeath M, Drysdale MTB, Bohn N. Work-integrated learning and the importance of peer support and sense of belonging. Educ Train. 2018;60(1):39-53. doi:10.1108/ET-05-2017-0070

29. Hawley LD, MacDonald MG, Wallace EH, Smith J, Wummel B, Wren PA. Baseline assessment of campus-wide general health status and mental health: opportunity for tailored suicide prevention and mental health awareness programming. $J$ Am Coll Heal. 2016;64(3):174-183. doi:10.1080/07448481.2015.1085059

30. Dinh KT, Holmberg MD, Ho IK, Haynes MC. The relationship of prejudicial attitudes to psychological, social, and physical wellbeing within a sample of college students in the United States. $J$ Cult Divers. 2013;21(2):56-66.

31. Meister SR, Barker B, Flores Pajot M-C. Heavy Episodic Drinking Among Post-
Secondary Students: Influencing Factors and Implications. Ottawa: Canadian Centre on Substance Use and Addiction; 2018. http://www.ccsa.ca.

32. Edwards K, Jones S. "Putting My Man Face On": a grounded theory of college men's gender identity development. $J$ Coll Stud Dev. 2007;50(2):210-228. doi:10.1353/csd.0.0063

33. Richardson T, Elliott P, Roberts R, Jansen M. A longitudinal study of financial difficulties and mental health in a national sample of British undergraduate students. Community Ment Health J. 2017;53(3):344352. doi:10.1007/s10597-016-0052-0

34. Walsemann KM, Gee GC, Gentile D. Sick of our loans: student borrowing and the mental health of young adults in the United States. Soc Sci Med. 2015;124:85-93. doi:10.1016/j.socscimed.2014.11.027

35. Welle PD, Graf HM. Effective lifestyle habits and coping strategies for stress tolerance among college students. $\mathrm{Am} \mathrm{J}$ Heal Educ. 2011;42(2):96-105. doi:10.1080/19325037.2011.10599177

36. Ridner SL, Newton KS, Staten RR, Crawford TN, Hall LA. Predictors of wellbeing among college students. $J$ Am Coll Heal. 2016;64(2):116-124. doi:10.1080/07448481.2015.1085057

37. Wyatt T, Oswalt SB. Comparing mental health issues among undergraduate and graduate students. Am J Heal Educ. 2013;44(2):96-107. doi:10.1080/19325037.2013.764248

38. Manderscheid RW, Ryff CD, Freeman EJ, McKnight-Eily LR, Dhingra S, Strine TW. Evolving definitions of mental illness and wellness. Prev Chronic Dis. 2010;7(1).

39. Miller DN, Gilman R, Martens MP. Wellness promotion in the schools: enhancing students' mental and physical health. Psychol Sch. 2008;45(1):5-15. doi: $10.1002 /$ pits. 20274 


\section{Appendix A}

Table A-1. Severity and Frequency Ratings for Each Stressor, by Proportion Reporting

\begin{tabular}{|c|c|c|c|c|c|c|}
\hline \multirow{2}{*}{ Stressor ID } & \multicolumn{3}{|c|}{ Female } & \multicolumn{3}{|c|}{ Male } \\
\hline & Severity & Frequency & Percent & Severity & Frequency & Percent \\
\hline \multicolumn{7}{|l|}{ Academic Domain } \\
\hline Exams (Prep) & 2.77 & 2.51 & 0.92 & 2.47 & 2.16 & 0.77 \\
\hline Exams (General) & 2.81 & 2.46 & 0.87 & 2.52 & 2.20 & 0.74 \\
\hline Exams (Multiple) & 3.47 & 2.16 & 0.66 & 3.14 & 1.72 & 0.67 \\
\hline Exams (Heavy) & 3.26 & 2.40 & 0.78 & 2.76 & 2.12 & 0.70 \\
\hline Assignments (Heavy) & 2.75 & 2.53 & 0.91 & 2.46 & 2.22 & 0.80 \\
\hline Assignments (Multiple) & 3.09 & 2.89 & 0.96 & 2.90 & 2.48 & 0.89 \\
\hline Workload & 2.53 & 2.85 & 0.93 & 2.44 & 2.56 & 0.83 \\
\hline Bad grades & 3.08 & 2.36 & 0.91 & 2.81 & 2.02 & 0.79 \\
\hline GPA & 2.85 & 2.72 & 0.89 & 2.69 & 2.61 & 0.74 \\
\hline Thesis & 3.02 & 2.71 & 0.28 & 2.72 & 2.41 & 0.38 \\
\hline Placement & 2.61 & 2.24 & 0.38 & 2.60 & 2.25 & 0.34 \\
\hline \multicolumn{7}{|c|}{ Learning Environment Domain } \\
\hline Poor Communication & 2.68 & 2.15 & 0.80 & 2.36 & 1.93 & 0.74 \\
\hline Clarity & 2.88 & 2.26 & 0.90 & 2.63 & 2.04 & 0.83 \\
\hline Guidance & 2.71 & 2.11 & 0.86 & 2.43 & 1.90 & 0.79 \\
\hline Meet prof & 2.51 & 1.85 & 0.50 & 2.43 & 1.91 & 0.36 \\
\hline Meet Advisor Expectations & 2.60 & 2.28 & 0.30 & 2.42 & 2.05 & 0.33 \\
\hline Mentoring & 2.87 & 2.18 & 0.28 & 2.54 & 2.00 & 0.28 \\
\hline \multicolumn{7}{|l|}{ Campus Culture Domain } \\
\hline Lifestyle & 2.40 & 1.94 & 0.62 & 2.42 & 1.87 & 0.45 \\
\hline Program & 2.40 & 2.09 & 0.71 & 2.28 & 2.06 & 0.60 \\
\hline Competition & 2.58 & 2.27 & 0.61 & 2.50 & 2.32 & 0.55 \\
\hline Work hard & 2.86 & 2.79 & 0.88 & 2.73 & 2.71 & 0.76 \\
\hline Smarter & 2.86 & 2.73 & 0.80 & 2.63 & 2.42 & 0.60 \\
\hline Succeed & 2.99 & 2.96 & 0.92 & 2.68 & 2.59 & 0.84 \\
\hline Discrim & 2.43 & 1.71 & 0.33 & 2.34 & 1.66 & 0.27 \\
\hline Sexual Harrass & 2.62 & 1.70 & 0.32 & 2.88 & 1.62 & 0.12 \\
\hline
\end{tabular}




\begin{tabular}{|c|c|c|c|c|c|c|}
\hline \multicolumn{7}{|l|}{ Interpersonal Domain } \\
\hline New Friends & 2.52 & 2.33 & 0.64 & 2.46 & 2.03 & 0.46 \\
\hline Maintain Friends & 2.41 & 2.39 & 0.59 & 2.30 & 2.11 & 0.50 \\
\hline Networking & 2.61 & 2.26 & 0.67 & 2.42 & 2.09 & 0.56 \\
\hline Social Pressure & 2.47 & 2.39 & 0.67 & 2.43 & 2.14 & 0.58 \\
\hline Balance (Social) & 2.60 & 2.61 & 0.82 & 2.36 & 2.48 & 0.72 \\
\hline Comparing & 2.81 & 2.71 & 0.83 & 2.61 & 2.53 & 0.68 \\
\hline Soc Media & 2.66 & 2.51 & 0.62 & 2.50 & 2.34 & 0.38 \\
\hline Meet Expectations (Other) & 2.72 & 2.57 & 0.78 & 2.53 & 2.35 & 0.59 \\
\hline Meet Expectations (self) & 3.10 & 3.10 & 0.92 & 2.85 & 2.93 & 0.89 \\
\hline \multicolumn{7}{|l|}{ Personal Domain } \\
\hline Sleep & 2.64 & 2.85 & 0.81 & 2.33 & 2.52 & 0.76 \\
\hline Exercise & 2.62 & 2.76 & 0.87 & 2.36 & 2.47 & 0.69 \\
\hline Nutrition & 2.60 & 2.77 & 0.83 & 2.34 & 2.42 & 0.69 \\
\hline Cooking & 2.56 & 2.63 & 0.64 & 2.34 & 2.47 & 0.49 \\
\hline Balance (Work) & 2.78 & 2.66 & 0.49 & 2.52 & 2.52 & 0.33 \\
\hline Balance (Extracurriculars) & 2.60 & 2.52 & 0.67 & 2.48 & 2.44 & 0.62 \\
\hline Hobbies & 2.72 & 2.65 & 0.73 & 2.45 & 2.35 & 0.64 \\
\hline Loans & 3.10 & 2.91 & 0.49 & 2.79 & 2.53 & 0.40 \\
\hline Debt & 3.13 & 2.90 & 0.55 & 2.67 & 2.56 & 0.51 \\
\hline Job & 3.08 & 2.79 & 0.83 & 2.92 & 2.73 & 0.69 \\
\hline New Program & 3.13 & 2.84 & 0.62 & 2.78 & 2.69 & 0.42 \\
\hline Life Events & 2.96 & 2.64 & 0.73 & 2.71 & 2.36 & 0.60 \\
\hline
\end{tabular}

Note. Percents indicate the proportion of respondents who experienced each stressor, calculated as the number of respondents who indicated that a stressor was 'somewhat stressful,' 'very stressful,' or 'extremely stressful,' divided by the total number of respondents to that question. Those who responded 'not applicable' or 'not stressful' were considered to be unaffected by the stressor." 


\section{Appendix B}

Table B-1. T-tests for Difference of Means, by Sex

\begin{tabular}{lcccccc}
\hline \multirow{2}{*}{ Question } & \multicolumn{3}{c}{ Severity } & \multicolumn{3}{c}{ Frequency } \\
\cline { 2 - 8 } ACADEMICS & $\mathbf{t}$ & $\mathbf{p}$ value & & $\mathbf{t}$ & p value \\
\hline Exams (Preparation) & & & & & & \\
Exams (General) & -3.9891 & 0.0001 & $*$ & -3.7736 & 0.0002 & $*$ \\
Exams (Multiple) & -3.4176 & 0.0008 & $*$ & -2.6712 & 0.0083 & $*$ \\
Exams (Heavy) & -3.4796 & 0.0007 & $*$ & -3.0951 & 0.0023 & $*$ \\
Assignments (Heavy) & -5.4879 & 0.0000 & $*$ & -2.1035 & 0.0372 & $*$ \\
Assignments (Multiple) & -4.0822 & 0.0001 & $*$ & -3.4922 & 0.0006 & $*$ \\
Workload & -2.6316 & 0.0091 & $*$ & -4.5774 & 0.0000 & $*$ \\
Bad grades & -1.3092 & 0.1921 & & -3.1674 & 0.0018 & $*$ \\
GPA & -3.1956 & 0.0017 & $*$ & -3.2538 & 0.0014 & $*$ \\
Thesis & -1.8911 & 0.0604 & & -1.0252 & 0.3070 & \\
Placement & -2.5033 & 0.0138 & $*$ & -1.8026 & 0.0746 & \\
\hline
\end{tabular}

LEARNING ENVIRONMENT

\begin{tabular}{lllllll}
\hline Poor Communication & -4.6133 & 0.0000 & $*$ & -2.2786 & 0.0240 & $*$ \\
Clarity & -3.2458 & 0.0014 & $*$ & -2.4942 & 0.0135 & $*$ \\
Guidance & -3.8221 & 0.0002 & $*$ & -2.0459 & 0.0425 & $*$ \\
Meet prof & -0.7829 & 0.4361 & & 0.4435 & 0.6587 & \\
Meet Advisor Expectations & -1.5192 & 0.1322 & & -1.4445 & 0.1524 & \\
Mentoring & -2.4098 & 0.0185 & $*$ & -0.9043 & 0.3694 \\
\hline
\end{tabular}

CAMPUS CULTURE

\begin{tabular}{|c|c|c|c|c|c|c|}
\hline Lifestyle & 0.1813 & 0.8565 & & -0.6351 & 0.5270 & \\
\hline Program & -1.8928 & 0.0602 & & -0.2760 & 0.7830 & \\
\hline Competition & -0.8746 & 0.3835 & & 0.4476 & 0.6552 & \\
\hline Work hard & -1.6070 & 0.1099 & & -0.8116 & 0.4181 & \\
\hline Smarter & -2.6744 & 0.0084 & $*$ & -2.8168 & 0.0056 & * \\
\hline Succeed & -3.7790 & 0.0002 & $*$ & -3.7462 & 0.0002 & * \\
\hline Discrim & -0.7471 & 0.4582 & & -0.2807 & 0.7801 & \\
\hline Sexual Harrass & 1.2177 & 0.2388 & & -0.2409 & 0.8125 & \\
\hline
\end{tabular}


INTERPERSONAL

\begin{tabular}{lllllll}
\hline New Friends & -0.6819 & 0.4968 & & -2.5096 & 0.0136 & $*$ \\
Maintain Friends & -1.3421 & 0.1821 & & -2.4064 & 0.0178 & $*$ \\
Networking & -2.5507 & 0.0117 & $*$ & -1.6675 & 0.0977 & \\
Social Pressure & -0.4114 & 0.6814 & & -2.3033 & 0.0229 & $*$ \\
Balance (Social) & -3.4018 & 0.0008 & $*$ & -1.3656 & 0.1740 & \\
Comparing & -2.3483 & 0.0201 & $*$ & -1.5512 & 0.1233 \\
Soc Media & -1.5186 & 0.1329 & & -1.1596 & 0.2500 & \\
Meet Expectations (Other) & -2.2431 & 0.0266 & $*$ & -2.0647 & 0.0409 & $*$ \\
Meet Expectations (self) & -3.0798 & 0.0024 & $*$ & -1.8605 & 0.0643 & \\
\hline
\end{tabular}

PERSONAL

\begin{tabular}{lllllll}
\hline Sleep & -4.1842 & 0.0000 & $*$ & -3.6029 & 0.0004 & $*$ \\
Exercise & -3.4650 & 0.0007 & $*$ & -2.7800 & 0.0062 & $*$ \\
Nutrition & -3.4256 & 0.0008 & $*$ & -3.2188 & 0.0016 & $*$ \\
Cooking & -2.5364 & 0.0124 & $*$ & -1.4885 & 0.1392 & \\
Balance (Work) & -2.1550 & 0.0346 & $*$ & -1.0139 & 0.3141 & \\
Balance (Extracurriculars) & -1.5535 & 0.1222 & & -0.8125 & 0.4178 & \\
Hobbies & -3.3098 & 0.0012 & $*$ & -2.9504 & 0.0037 & $*$ \\
Loans & -2.5159 & 0.0137 & $*$ & -2.5047 & 0.0142 & $*$ \\
Debt & -4.4213 & 0.0000 & $*$ & -2.5159 & 0.0134 & $*$ \\
Job & -1.6464 & 0.1018 & & -0.4857 & 0.6279 & \\
New Program & -2.8969 & 0.0049 & $*$ & -1.0921 & 0.2780 & \\
Life Events & -2.4542 & 0.0154 & $*$ & -2.3037 & 0.0229 & $*$
\end{tabular}




\section{Appendix C}

Stressors items were shortened into stressor IDs to facilitate a clear display on the plots throughout this article. A reference codebook follows, identifying the items the IDs represent.

Table C-1. PSSI Codebook

\begin{tabular}{|c|c|}
\hline Stressor ID & PSSI Item \\
\hline \multicolumn{2}{|l|}{ Academics } \\
\hline Exams (Preparation) & Preparing for exams \\
\hline Exams (General) & Writing exams \\
\hline Exams (Multiple) & Writing multiple exams same day \\
\hline Exams (Heavy) & Writing exams worth $50 \%$ or more \\
\hline Assignments (Heavy) & Heavily weighted assignments \\
\hline Assignments (Multiple) & Having multiple assignments due around the same time \\
\hline Workload & Managing my academic workload \\
\hline Bad grades & Receiving a bad grade \\
\hline GPA & Maintaining a high GPA \\
\hline Thesis & Working on my thesis \\
\hline Placement & Performing well at my placement (i.e., practicum) \\
\hline \multicolumn{2}{|l|}{ Learning Environment } \\
\hline Poor Communication & Poor communication from professor \\
\hline Clarity & Unclear expectations from professor \\
\hline Guidance & Lack of guidance from professor \\
\hline Meet prof & Meeting with professor \\
\hline Meet Advisor Expectations & Meeting my supervisor's expectations (placement/thesis) \\
\hline Mentoring & Lack of mentoring from my supervisor (placement/thesis) \\
\hline \multicolumn{2}{|l|}{ Campus Culture } \\
\hline Lifestyle & Adjusting to the post-secondary lifestyle \\
\hline Program & Adjusting to my program \\
\hline Competition & Academic competition among my peers \\
\hline Work hard & Feeling like I'm not working hard enough \\
\hline Smarter & Feeling like my peers are smarter than I am \\
\hline Succeed & Pressure to succeed \\
\hline Discrim & Discrimination on campus \\
\hline Sexual Harrass & Sexual harassment on campus \\
\hline
\end{tabular}




\begin{tabular}{ll}
\hline Interpersonal & Making new friends \\
New Friends & Maintaining friendships \\
Networking & Networking with the 'right' people \\
Social Pressure & Feeling pressured to socialize \\
Balance (Social) & Balancing a social life with my academics \\
Comparing & Comparing myself to others \\
Soc Media & Comparing my life to others on social media \\
Meet Expectations (Other) & Meeting other peoples' expectations of me \\
Meet Expectations (self) & Meeting my own expectations \\
\hline Personal & \\
\hline Sleep & Getting enough sleep \\
Exercise & Getting enough exercise \\
Nutrition & Making sure I eat healthy \\
Cooking & Having to prepare meals for myself \\
Balance (Work) & Balancing working at my job with academics \\
Balance (Extracurriculars) & Balancing my extracurriculars with academics \\
Hobbies & Feeling guilty about taking time for my hobbies/interests \\
Loans & Having to take student loans \\
Debt & Worrying about paying off debt \\
Job & Worrying about getting a job after graduation \\
New Program & Worrying about getting in to a new program after graduation \\
Life Events & Worrying about meeting major life events (e.g., marriage) \\
\hline & \\
\hline &
\end{tabular}

